Báez Mirón, F.; Zurita Ortega, F.; Martínez Martínez, A. y Zagalaz Sánchez, M.L. (2019). Análisis psicométrico y relaciones de diagnóstico de la inteligencia emocional y liderazgo en docentes de enseñanzas regladas. Revista de Investigación Educativa, 37(1), 201-216.

DOI: http://dx.doi.org/10.6018/rie.37.1.308801

\title{
Análisis psicométrico y relaciones de diagnóstico de la inteligencia emocional y liderazgo en docentes de enseñanzas regladas
}

\section{Psychometric analysis and diagnosis relationships of emotional intelligence and leadership in teachers of regulated education}

\author{
Federico Báez Mirón*, Félix Zurita Ortega**, \\ Asunción Martínez Martínez** y Ma ${ }^{\text {a }}$ Luisa Zagalaz Sánchez* \\ *Universidad de Jaén \\ **Universidad de Granada
}

\begin{abstract}
Resumen
La inteligencia emocional y el liderazgo son dos elementos básicos en las competencias y habilidades de los docentes. En este estudio que tiene como objetivos comprobar las propiedades psicométricas de TMSS-24 y MLQ-5X en docentes, describir la competencia de inteligencia emocional y de liderazgo de éstos, y establecer las relaciones de éstas con el tipo de enseñanza que se imparte. Para ello se analizaron a 160 docentes de toda España de 137 centros educativos, a los cuales les fue administrado los instrumentos anteriormente mencionados. Se debe indicar como principales resultados el excelente ajuste de estos instrumentos así como su fiabilidad siendo adecuadas para el conjunto de docentes de enseñanzas regladas. Dentro del marco de la inteligencia emocional se debe señalar que la regulación y comprensión son las más valoradas y en cuanto al liderazgo los de tipos transformacionales, transaccionales y resultados de liderazgo presentan cifras muy similares. En el ámbito de las relaciones solamente se encontró asociación entre el liderazgo transformacional y el tipo de enseñanza impartida hallándose una mayor capacidad de cambio en el contexto universitario, mientras que en primaria y ciclos formativos la transformación no es tan importante.

Palabras clave: psicométrico; liderazgo; inteligencia emocional; docentes.
\end{abstract}

Correspondencia: Félix Zurita Ortega, felixzo@ugr.es, Facultad de Ciencias de la Educación, Universidad de Granada, Campus Universitario de Cartuja, Granada (18071) España. 


\begin{abstract}
Emotional intelligence and leadership are two basic elements in the competences and abilities of teachers. In this study, which aims to verify the psychometric properties of TMSS-24 and $M L Q-5 X$ in teachers, describe the competence of emotional intelligence and leadership of these, and establish the relationships of these with the type of teaching that is taught. To this end, 160 teachers from all over Spain were analyzed from 137 educational centers, to which the aforementioned instruments were administered. It should be indicated as the main results the excellent adjustment of these instruments as well as their reliability being fully adequate for the group of teachers of regulated education. Within the framework of emotional intelligence should be noted that regulation and understanding are the most valued and in terms of leadership transformational types, transactional and leadership results have very similar figures. In the field of relationships, only an association was found between the transformational leadership and the type of education given, with a greater capacity for change in the university context, while in primary and vocational training the transformation is not so important.

Keywords: psychometrics; leadership; intelligence emotional; teachers.
\end{abstract}

\title{
Introducción
}

No cabe duda que el hecho de considerar a la Inteligencia emocional (IE) en el siglo XXI como complemento del desarrollo cognitivo que facilita las relaciones en los centros de trabajo, y por tanto en las instituciones docentes como mejora de las interacciones entre los miembros de cualquier comunidad, educativa o no, se puede considerar de vital importancia para el óptimo funcionamiento de éstas (Cabello, Ruiz \& Berrocal, 2010).

Al hilo de esto y siguiendo las palabras de Mayer y Salovey (1997, p. 10) se puede considerar la IE como un elemento didáctico, ya que definían la IE como:

la capacidad para percibir, valorar y expresar las emociones con exactitud; la capacidad para acceder y generar sentimientos que faciliten el pensamiento; la capacidad para entender la emoción y el conocimiento emocional; y la capacidad para regular las emociones y promover el crecimiento emocional e intelectual.

Enraizado con el anterior planteamiento, Palomo-Vadillo (2013) argumenta que en todas las organizaciones sujetas a procesos de cambios constantes, los líderes se convierten en una pieza clave que completa el complicado puzle que se va planteando y permite que todas las piezas encajen perfectamente, sin olvidar otros elementos clave como los recursos humanos, los procesos y la estructura como organización. Para ello, los líderes tienen que ser capaces de:

- Anticipar y tener una visión futura de la realidad, siendo proactivo y previsor de forma permanente.

- Adquirir y desarrollar competencias críticas para gestionar los cambios en la institución. 
- Participar y potenciar el aprendizaje continuo, tanto el propio como el de sus colaboradores, facilitando el poder guiar y orientar a éstos en el cambio.

- Facilitar y estimular el desarrollo del potencial y de las competencias de su equipo.

- Implicar y hacer participar a todo el personal de la institución, derribando, en algunos casos barreras culturales y actitudinales del personal que gestiona.

El interés por comprender las dinámicas internas de los cargos docentes y de dirección responde tanto a cuestiones de orden institucional como de tipo personal. Institucionalmente estos puestos representan los niveles estratégicos de la organización y desarrollan un rol fundamental en la estrategia de centros y departamentos. En cuanto al desarrollo de las personas y su carrera profesional nos brinda la posibilidad de intervenir sobre estos puestos específicos permitiendo una mayor coherencia y una mejor articulación entre las funciones asignadas (Castro \& Tomás, 2010; Ritacco \& Bolívar, 2016; Ritacco-Real \& Amores-Fernández, 2017).

El aprendizaje de conocimientos, procedimientos, actitudes y valores, se ve favorecido cuando los profesores se hacen conscientes de los hábitos que tiene el alumnado, explicitándose en la planificación las actitudes que se pretende que adquieran y seleccionándose aquellas actividades y estrategias de enseñanza adecuadas para favorecer el desarrollo actitudinal. Además de realizar una cuidadosa selección de los objetivos y contenidos de aprendizaje, se hace necesario planificar para cada tipo de enseñanza y nivel educativo la estrategia que más se adecue al desarrollo de nuestros alumnos en relación con el contexto de cada comunidad de aprendizaje para que proporcione suficientes oportunidades con el fin de que las pongan en práctica en diferentes situaciones educativas (Estela, Gálvez, Del Ángel, Mejía \& Rodiles, 2013).

La vigencia del liderazgo y la educación como tema de investigación se muestra en un gran conjunto de publicaciones, que han ido en aumento en la última década (ÁlvarezBotello, Torres-Velázquez \& Chaparro-Salinas, 2016; Argos \& Ezquerra, 2013; Bolívar, López \& Murillo, 2013; Harris, 2009; Hernández-Castilla, Murillo, e Hidalgo, 2017; Leithwood \& Louis, 2011; Scheerens, 2012; MacBeath \& Cheng, 2008; MacBeath \& Townsend, 2011; Muri1lo \& Hernández-Castilla, 2015; Puertas-Molero, González-Valero \& Sánchez-Zafra, 2017).

Así, al ser el liderazgo pedagógico uno de los medios fundamentales para mejorar la calidad educativa, se propone considerar el liderazgo del profesor como uno de los principales cometidos en la gestión de los centros educativos, diseñando programas de formación. Numerosos estudios son ejemplo de este planteamiento, como los de Daly y Finnigan (2016); Elfers y Stritikus (2014); Hué, Esteban y Bardisa (2012); RuizCorbella (2013). Sin embargo, el interés radica en comprender cómo un docente puede ejercer un liderazgo propio, como profesor.

Al analizarse la situación del profesor para ser líder (Bisquerra-Alzina, 2003; Frost \& Harris, 2003; Robinson, 2010), se observan tres aspectos que aparecen en la bibliografía de manera redundante:

- La concepción de la enseñanza que tiene el profesor.

- Su competencia emocional.

- Su percepción acerca del clima socioemocional del centro educativo. 
Por otro lado, otro parámetro importante en el estudio del liderazgo del profesor es la competencia emocional (Cazalla-Luna \& Molero, 2016; Day, 2012; Hargreaves, 2002; Hagreaves \& Fullan,2014; Zembylas \& Schutz, 2009), a ello se suma la entrega al trabajo a través de la oportunidad de participación en las diferentes tareas, proyectos o actividades, como reflejo de dicho compromiso docente, y también para el logro de fines conjuntos en la comunidad educativa (Tichy, 2003).

Duarte-Rodríguez (2016) aporta que otro factor que se ha definido como relevante para la función de dirección y del cargo docente es la IE del gerente. La importancia sobre este tema surge a raíz de ese conjunto de habilidades que, se supone, son pilares en el desempeño del equipo directivo, puesto que se considera que la IE es un conjunto de habilidades entre las que se destacan el autocontrol, el entusiasmo, la empatía, la perseverancia y la capacidad para motivarse a uno mismo (Goleman, 2012). Lo que a su vez hace un individuo emocionalmente equilibrado apto para el cargo docente que ostente en la institución educativa que desempeña su labor.

Murillo y Hernández-Castilla (2015) ahondan en la organización del tiempo y las tareas que desempeñan cargo docentes directivos de las escuelas de Educación Primaria que fomentan el aprendizaje de los estudiantes en España utilizando la base de datos de la Evaluación General de Diagnóstico de Educación Primaria desarrollada en España en 2009, utilizando Modelos Multinivel.

En España destacan dos trabajos. Por un lado, el estudio de Murillo y Barrio (1999) con datos de 437 directores y directoras de Educación Primaria. Y, por otro, un reciente e interesante estudio desarrollado por López-Yáñez, García-Jiménez, Oliva-Rodríguez, MoretaJurado y Bellerín (2014) sobre la actividad diaria de los directores y directoras de escuela.

\section{Método}

\section{Objetivos del Estudio}

Por tanto en este estudio se aporta como dato novedoso la importancia que tiene la inteligencia emocional en la capacidad de liderazgo del docente, así como la implementación de dos instrumentos para su análisis, para ello se plantea este trabajo con los objetivos de comprobar las propiedades psicométricas de TMSS-24 y MLQ-5X en docentes, describir la competencia de inteligencia emocional y de liderazgo de éstos, y establecer las relaciones de éstas con el tipo de enseñanza que imparten los profesores.

\section{Población y Muestra}

Participaron en ésta investigación de carácter descriptivo y de tipo transversal un total de 160 docentes de toda España, de ambos sexos (45.6\% hombres y $54.4 \%$ mujeres) pertenecientes a 137 centros educativos de diversa tipología de enseñanza.

En lo referente a la enseñanza donde se imparten, la mayoría de los profesores proceden de la Universidad (28.7\%), seguido de los de Primaria (25.6\%) y Secundaria (23.8\%), por el contrario los de Infantil, Ciclos Formativos y Otros, no alcanzan el 10\% de los casos, también indicar que el 37.5\% tienen entre 30 y 39 años y el $27.5 \%$ entre 40 y 49 años, por el contrario solamente un $16 \%$ son docentes con más de 50 años (ver en tabla 3 ). 
La muestra se obtuvo mediante un muestreo aleatorio estratificado del profesorado de todos los niveles educativos que se imparten en España, incluido el universitario. Se debe indicar que se detectaron 36 cuestionarios erróneos en su realización que fueron excluidos del estudio.

\section{Instrumento}

Para la presente investigación se han utilizado tres tipos de instrumentos, el primero de ellos es un cuestionario $u$ hoja de Autorregistro donde el docente indicaba su género, rango de edad y tipo de enseñanza que se impartía, ya fuese Infantil, Primaria, Secundaria, Ciclos Formativos, Universidad y Otros (donde se aglutinaban docentes de Religión, Orientadores, Educación Especial, Educación de Adultos...).

El segundo instrumento es el MLQ-5x de Bass y Avolio (1999) denominado en su versión original como (Multifactor Leadership Questionnaire), es considerado como "el instrumento más popular para medir el liderazgo transformacional" (Antonakis \& House, 2013). Consta de 45 ítems, medidos a través de una escala Likert de cinco opciones de respuesta donde el 0 es "Nada en absoluto" y el 4 es "Muy deacuerdo", los primeros 36 ítems identifican las conductas de liderazgo en los líderes (Transformacional, Transaccional y laissez-faire) mientras que los ítems 37 a 45 responde a los resultados de liderazgo. En el contexto español en su validación realizada por Molero-Alonso, RecioSaboya y Cuadrado-Guirado (2010) se obtiene un Alpha de Cronbach superior al .700.

El tercer instrumento empleado es el TMMS-24 extraído del Trait Meta-Mood Scale (TMMS) del grupo de investigación de. Salovey, Mayer, Goldman, Turvey y Palfai (1995). La escala original evalúa el metaconocimiento de los estados emocionales mediante 24 ítems, estructurado en tres dimensiones (percepción, comprensión y regulación) con 8 ítems cada una de ellas respondidos mediante una escala Likert de 5 puntos; percepción emocional, comprensión de sentimientos y regulación emocional.

En el contexto nacional ha sido empleado por Cazalla-Luna y Molero (2016) que señalan valores superiores al .700 según Alpha de Cronbach.

En concreto, las destrezas con las que podemos ser conscientes de nuestras propias emociones, así como de nuestra capacidad para regularlas.

\section{Procedimiento de recogida y análisis de datos}

Los cuestionarios se administraron en horario externo a las horas de clase y se aplicaron de manera individual. Se garantizó a los participantes el anonimato de la información recogida y aclarando que su utilización sería sólo con fines científicos para su posterior difusión. Los participantes desconocen la finalidad del estudio con el fin de evitar respuestas no sinceras y reducir al máximo posible el efecto de deseabilidad social.

En este estudio, las propiedades psicométricas (fiabilidad y análisis factorial exploratorio) se realizan mediante el Programa FACTOR Analysis 9.3.1 (Lorenzo-Seva y Ferrando, 2006) y el análisis descriptivo y relacional mediante el paquete estadístico SPSS 23.0. En primer lugar se emplea el SPSS 23.0., para analizar las propiedades métricas de cada ítem y para ello se emplearon los coeficientes descriptivos básicos (media, dispersión, curtosis y asimetría). En segundo lugar se utiliza el FACTOR, 
donde el diagnóstico de la bondad de ajuste es esencial para establecer la validez de la escala. La evaluación se ha basado en varios criterios como recomiendan Bentler (1990) y McDonald y Marsh (1990). Con la finalidad de verificar y determinar la consistencia interna del instrumento y de las distintas dimensiones se usó el coeficiente alpha de Cronbach, para finalizar se usaron los valores descriptivos y las ANOVA para el estudio relacional, todo ellos a través del SPSS 23.0.

\section{Resultados}

En esta primera parte del análisis se procede a determinar el Análisis Factorial Exploratorio (AFE) de los cuestionarios TMSS-24 y MLQ-5X. En primer lugar, y respecto al TMSS-24 no se elimina ninguna variable como planteaban los expertos (Schmider, Ziegler, Danay, Beyer \& Bühner, 2010) que señalaban la posibilidad de prescindir de alguna dimensión o variable cuando se presentasen cifras superiores a 2.00 en las pruebas de dispersión como son la asimetría y la curtosis. Seguidamente, mediante la utilización del programa FACTOR Analysis (Lorenzo-Seva \& Ferrando, 2006) se ha comenzado a desarrollar el AFE, para ello se han rotado tres factores. El estadístico de Bartlett, [2045.1 ( $\mathrm{df}=276 ; p=.000010)]$ y el test de Kaiser-Meyer-Olkin $(\mathrm{KMO})=.845$, utilizados para probar si la muestra procede de poblaciones con la misma varianza y si presenta una buena adecuación muestral, indican un buen ajuste de los datos para ser sometidos al análisis factorial. Los tres factores extraídos explican el $55 \%$ de la varianza total, el índice de bondad de ajuste (GFI) fue de .98, el índice ajustado de bondad de ajuste (AGFI) de .97 y la raíz cuadrática media de los residuales (RMSR) de .04. Todos estos datos nos indican un buen ajuste para estos ítems de este cuestionario.

Como se determina en la siguiente tabla 1 se ha eliminado la variable (V 23), por presentar valores menores a .300. La escala definitiva ha quedado formada por tres factores, el primero de ellos se encuentra conformado por ocho variables y se corresponde con la dimensión comprensión; un segundo factor constituido por ocho variables se relaciona con la dimensión percepción; y por último un tercer factor constituido por siete variables que se corresponde con la dimensión regulación.

A continuación se muestra la configuración del cuestionario con un buen ajuste, puesto que el coeficiente de fiabilidad del cuestionario fue de .860 , y suprimidas las variables que no alcanzaban las directrices, por factores se obtuvo un .870 para el factor 1 (comprensión), .896 para el factor 2 (percepción), y .850 en el factor 3 (regulación).

Tabla 1

Carga factorial de las dimensiones del test de inteligencia emocional (TMSS-24)

\begin{tabular}{cccc}
\hline Variables & F1 & F2 & F3 \\
\hline V 09 & .561 & & \\
V 10 & .847 & & \\
V 11 & .809 & & \\
V 12 & .777 & & \\
V 13 & .559 & &
\end{tabular}




\begin{tabular}{|c|c|c|c|}
\hline V 14 & .745 & & \\
\hline V 15 & .550 & & \\
\hline V 16 & .651 & & \\
\hline V 01 & & .634 & \\
\hline V 02 & & .765 & \\
\hline V 03 & & .744 & \\
\hline V 04 & & .667 & \\
\hline V 05 & & .634 & \\
\hline V 06 & & .703 & \\
\hline V 07 & & .750 & \\
\hline V 08 & & .784 & \\
\hline V 17 & & & .761 \\
\hline V 18 & & & .853 \\
\hline V 19 & & & .626 \\
\hline V 20 & & & .840 \\
\hline V 21 & & & .593 \\
\hline V 22 & & & .531 \\
\hline V 24 & & & .617 \\
\hline Alfa (.860) & .870 & .896 & .850 \\
\hline
\end{tabular}

En segundo lugar, se realiza el AFE del MLQ-5X, siguiendo las premisas de Schmider et al. (2010), cuando señalaba que los valores de dispersión (asimetría y curtosis) deben ser inferiores a 2.00, se aprecia como la variable número siete supera ese valor por lo que se prescinde de la misma, aplicándose el programa FACTOR Analysis (Lorenzo-Seva \& Ferrando, 2006) se han rotado cuatro factores. El estadístico de Bartlett, [3280.1 ( $\mathrm{df}=$ 990; $p=.000010)$ ] y el test de Kaiser-Meyer-Olkin $(\mathrm{KMO})=.856$, utilizados para probar si la muestra procede de poblaciones con la misma varianza y si presenta una buena adecuación muestral, indican un buen ajuste de los datos para ser sometidos al análisis factorial. Los cuatro factores extraídos explican el $42 \%$ de la varianza total, el índice de bondad de ajuste (GFI) fue de .96, el índice ajustado de bondad de aju0ste (AGFI) de .96 y la raíz cuadrática media de los residuales (RMSR) de .057. Todos estos datos nos indican un buen ajuste para estos ítems.

Se han eliminado las variables V 02, V 10, V 18, V 19, V 21, V 23, V 34, V 35 y V 37 , por presentar valores menores de .300 . La escala definitiva ha quedado formada por cuatro factores. El primero conformado por ocho variables se corresponde con los resultados de liderazgo, un segundo factor formado por trece variables que se relaciona con el liderazgo transformacional, un tercer factor que se corresponde con el liderazgo transaccional y formado por once variables y un cuarto factor constituido por tres variables que se relaciona con el liderazgo pasivo o evitador.

A continuación se muestra la configuración del cuestionario con un buen ajuste, el coeficiente de fiabilidad del cuestionario fue de .855, y suprimidas las variables, por factores se obtuvo un .856 para el factor 1 (resultados de liderazgo), .849 para el factor 2 (liderazgo transformacional), .789 para el factor 3 (liderazgo transaccional) y .802 en el factor 4 (liderazgo pasivo o evitador) (ver tabla 2). 
Tabla 2

Carga factorial de las dimensiones del test de liderazgo MLQ-5X

\begin{tabular}{|c|c|c|c|c|}
\hline Variables & F1 & F2 & F3 & F4 \\
\hline V 38 & .646 & & & \\
\hline V 39 & .587 & & & \\
\hline V 40 & .544 & & & \\
\hline V 41 & .366 & & & \\
\hline V 42 & .685 & & & \\
\hline V 43 & .683 & & & \\
\hline V 44 & .816 & & & \\
\hline V 45 & .760 & & & \\
\hline V 06 & & .659 & & \\
\hline V 08 & & .392 & & \\
\hline V 09 & & .307 & & \\
\hline V 13 & & .779 & & \\
\hline V 14 & & .311 & & \\
\hline V 15 & & .582 & & \\
\hline V 25 & & .494 & & \\
\hline V 26 & & .535 & & \\
\hline V 29 & & -.369 & & \\
\hline V 30 & & .604 & & \\
\hline V 31 & & .615 & & \\
\hline V 32 & & .445 & & \\
\hline V 36 & & .447 & & \\
\hline V 01 & & & -.350 & \\
\hline V 03 & & & .595 & \\
\hline V 04 & & & -.505 & \\
\hline V 11 & & & .582 & \\
\hline V 12 & & & .508 & \\
\hline V 16 & & & .650 & \\
\hline V 17 & & & .488 & \\
\hline V 20 & & & .548 & \\
\hline V 22 & & & .343 & \\
\hline V 24 & & & .472 & \\
\hline V 27 & & & .432 & \\
\hline V 05 & & & & .564 \\
\hline V 28 & & & & .380 \\
\hline V 33 & & & & .431 \\
\hline Alfa $(0.855)$ & .856 & .849 & .798 & .802 \\
\hline
\end{tabular}


En la segunda parte del análisis y comprobada la excelente estructura psicométrica de los cuestionarios se realiza el análisis descriptivo de los mismos, indicándose como en el caso de la IE, la regulación es la opción más valorada $(\mathrm{M}=2.04$; $\mathrm{DT}=0.530)$, seguido de la comprensión ( $\mathrm{M}=1.97 ; \mathrm{DT}=0.565)$ y finalmente la percepción $(\mathrm{M}=1.81 ; \mathrm{DT}=0.585)$. En lo referente al MLQ-5X, el liderazgo de tipo transformacional, transaccional y resultados de liderazgo presentaron cifras similares (en torno al valor 3 de media), mientras que el liderazgo pasivo obtuvo cifras de 1 de media.

Tabla 3

Descriptivos de las variables académicas

\begin{tabular}{|c|c|c|c|c|}
\hline $\begin{array}{l}\text { Tipo de Enseñanza } \\
\qquad n=160\end{array}$ & $\begin{array}{c}\text { Masculino } \\
45.6 \%(n=73)\end{array}$ & $\begin{array}{c}\text { Femenino } \\
54.4 \%(n=87)\end{array}$ & \multicolumn{2}{|c|}{ Liderazgo } \\
\hline $\begin{array}{c}\text { Infantil } \\
6.9 \%(\mathrm{n}=11)\end{array}$ & $1.4 \%(\mathrm{n}=1)$ & $11.5 \%(\mathrm{n}=10)$ & Transformacional & $\begin{aligned} \mathrm{M} & =3.08 \\
\mathrm{DT} & =0.450\end{aligned}$ \\
\hline $\begin{array}{c}\text { Primaria } \\
25.6 \%(n=41)\end{array}$ & $24.7 \%(n=18)$ & $26.4 \%(n=23)$ & Transaccional & $\begin{aligned} \mathrm{M} & =2.93 \\
\mathrm{DT} & =0.484\end{aligned}$ \\
\hline $\begin{array}{c}\text { Secundaria } \\
23.8 \%(\mathrm{n}=38)\end{array}$ & $28.8 \%(n=21)$ & $19.5 \%(\mathrm{n}=17)$ & Pasivo o Evitador & $\begin{aligned} \mathrm{M} & =0.97 \\
\mathrm{DT} & =0.605\end{aligned}$ \\
\hline $\begin{array}{c}\text { Ciclos Formativos } \\
5.6 \%(\mathrm{n}=9)\end{array}$ & $6.8 \%(n=5)$ & $4.6 \%(n=4)$ & $\begin{array}{l}\text { Resultados de } \\
\text { Liderazgo }\end{array}$ & $\begin{aligned} \mathrm{M} & =2.95 \\
\mathrm{DT} & =0.579\end{aligned}$ \\
\hline $\begin{array}{l}\text { Universidad } \\
28.8 \%(\mathrm{n}=46)\end{array}$ & $35.6 \%(n=26)$ & $23.0 \%(n=20)$ & & \\
\hline $\begin{array}{c}\text { Otros } \\
9.4 \%(\mathrm{n}=15) \\
\end{array}$ & $2.7 \%(n=2)$ & $14.9 \%(n=13)$ & & \\
\hline $\begin{array}{c}\text { Rango de Edad } \\
n=160\end{array}$ & & & \multicolumn{2}{|c|}{ Inteligencia Emocional } \\
\hline $\begin{array}{c}\text { Menos de } 30 \text { años } \\
18.8 \%(\mathrm{n}=30)\end{array}$ & $15.1 \%(n=11)$ & $21.8 \%(n=19)$ & Percepción & $\begin{array}{c}\mathrm{M}=1.81 \\
\mathrm{DT}=0.585\end{array}$ \\
\hline $\begin{array}{c}\text { De } 30 \text { a } 39 \text { años } \\
37.5 \%(n=60)\end{array}$ & $28.8 \%(n=21)$ & $44.8 \%(n=39)$ & Comprensión & $\begin{aligned} \mathrm{M} & =1.97 \\
\mathrm{DT} & =0.565\end{aligned}$ \\
\hline $\begin{array}{c}\text { De } 40 \text { a } 49 \text { años } \\
27.5 \%(\mathrm{n}=44) \\
\end{array}$ & $34.2 \%(\mathrm{n}=25)$ & $21.8 \%(\mathrm{n}=19)$ & Regulación & $\begin{aligned} \mathrm{M} & =2.04 \\
\mathrm{DT} & =0.530\end{aligned}$ \\
\hline $\begin{array}{c}\text { De } 50 \text { a } 59 \text { años } \\
10.0 \%(\mathrm{n}=16)\end{array}$ & $12.3 \%(n=9)$ & $8.0 \%(\mathrm{n}=7)$ & & \\
\hline $\begin{array}{c}\text { Más de } 60 \text { años } \\
6.3 \%(\mathrm{n}=10)\end{array}$ & $9.6 \%(n=7)$ & $3.4 \%(n=3)$ & & \\
\hline
\end{tabular}

Por último se realiza el análisis mediante ANOVA, no apreciándose diferencias estadísticamente significativas en el caso de la inteligencia emocional en función del tipo de enseñanza en que se imparte docencia $(p \geq .05)$, obteniéndose valores muy similares, pese a ello los docentes de infantil obtienen mayor puntuación media en la percepción, en el caso de la comprensión son los categorizados como otros tipos de enseñanza y en regulación son los docentes universitarios, como se puede desprender de los resultados expuestos en la tabla 4 . 
Tabla 4

Relación de la inteligencia emocional con el tipo de enseñanza

\begin{tabular}{cccccc}
\hline \multicolumn{2}{c}{ Inteligencia Emocional } & Media & DT & F & $p$ \\
\hline & Infantil & 1.91 & 0.539 & & \\
& Primaria & 1.83 & 0.495 & & \\
Percepción Inteligencia & Secundaria & 1.84 & 0.679 & 0.275 & .927 \\
Emocional & Ciclos Formativos & 1.78 & 0.441 & & \\
& Universidad & 1.80 & 0.619 & & \\
& Otros & 1.67 & 0.617 & & \\
& Infantil & 2.09 & 0.539 & & \\
Comprensión Inteligencia & Primaria & 1.90 & 0.625 & & \\
Emocional & Secundaria & 1.89 & 0.559 & \multirow{2}{*}{0.626} & .680 \\
& Ciclos Formativos & 2.00 & 0.707 & & \\
& Universidad & 2.00 & 0.471 & & \\
& Otros & 2.13 & 0.640 & & \\
\hline Regulación Inteligencia & Infantil & 2.00 & 0.632 & & \\
Emocional & Primaria & 2.07 & 0.519 & & \\
& Secundaria & 1.97 & 0.592 & \multirow{2}{*}{0.462} & .804 \\
& Ciclos Formativos & 1.89 & 0.601 & & \\
\hline
\end{tabular}

Así mismo si se detectan diferencias estadísticamente significativas ( $p \leq .050)$, en el caso del liderazgo transformacional con el lugar donde se imparte docencia, estas proceden de los colectivos de docentes de Primaria y Ciclos Formativos que obtienen valores medios $(M=2.90)$ inferiores al resto de enseñanzas, sobre todo a los de Universidad y Otros con valores medios superiores al 3.22 como se desprende de la siguiente tabla 5 .

Tabla 5

Relación del liderazgo con el tipo de enseñanza

\begin{tabular}{lccccc}
\hline & & Media & DT & F & $p$ \\
\hline & Infantil & 3.05 & 0.482 & & \\
& Primaria & 2.92 & 0.443 & & \\
Liderazgo Transformacional & Secundaria & 3.04 & 0.475 & 2.983 & .013 \\
& Ciclos Formativos & 2.90 & 0.452 & & \\
& Universidad & 3.22 & 0.417 & & \\
& Otros & 3.27 & 0.307 & & \\
& Infantil & 3.05 & 0.510 & & \\
& Primaria & 2.80 & 0.468 & & \\
& Secundaria & 2.91 & 0.467 & \multirow{2}{*}{1.769} & .122 \\
& Ciclos Formativos & 2.73 & 0.501 & & \\
& Universidad & 3.06 & 0.465 & & \\
\hline
\end{tabular}




\begin{tabular}{lcllll}
\hline & Infantil & 0.90 & 0.550 & & \\
& Primaria & 0.96 & 0.663 & & \\
Liderazgo Pasivo o Evitador & Secundaria & 0.95 & 0.661 & 1.611 & .160 \\
& Ciclos Formativos & 1.16 & 0.690 & & \\
& Universidad & 1.08 & 0.528 & & \\
& Otros & 0.61 & 0.396 & & \\
& Infantil & 2.85 & 0.702 & & \\
& Primaria & 2.84 & 0.591 & & \\
Resultados de Liderazgo & Secundaria & 2.87 & 0.524 & \multirow{2}{*}{1.626} & .156 \\
& Ciclos Formativos & 2.82 & 0.751 & & \\
& Universidad & 3.09 & 0.569 & & \\
\hline & Otros & 3.17 & 0.417 & & \\
\hline
\end{tabular}

\section{Discusión y conclusiones}

La configuración del cuestionario TMSS-24 ha presentado un buen ajuste en sus propiedades psicométricas, esto coincide con lo aportado por Ortega-Álvarez, NúñezHergueta, Molero y Torres-González (2015) en un trabajo enmarcado en el ámbito de la educación de la inteligencia emocional y satisfacción vital de futuros docentes, los resultados obtenidos ponen de manifiesto que las dimensiones más consideradas por la muestra objeto de estudio, de manera global, han sido la comprensión y la regulación, poniendo de manifiesto su fiabilidad y validez, y ayudando a comprender las capacidades emocionales de los docentes españoles (Cazalla-Luna \& Molero, 2014).

En lo referente al MLQ-5X se apreció igualmente un excelente ajuste, obteniéndose cifras similares a las de los estudios de Molero-Alonso et al. (2010), que también mostraban otras posibles alternativas, para concluir como uno de los instrumentos más usados para medir el liderazgo en el campo de la Psicología de las Organizaciones (Avolio \& Bass, 2004), lo que indica la importancia de este instrumento para el análisis del liderazgo en docentes de distintas especialidades.

Los resultados revelan que el modelo que mejor ajuste presenta es el que está formado por cuatro factores: liderazgo transformacional, liderazgo facilitador del desarrollo/transaccional, liderazgo correctivo o por excepción activa y liderazgo pasivo/evitador. Por lo que éste modelo es parsimonioso y teóricamente coherente con nuestro estudio.

En el caso de la inteligencia emocional, la regulación es la opción más valorada, seguido de la comprensión y finalmente la percepción, ello es debido a que el autoconocimiento nos permite controlar y gestionar, por tanto, nuestras emociones y establecer prácticas saludables que mejoren nuestra salud y calidad de vida. Es por ello por lo que se hace imprescindible que los docentes enseñen técnicas y destrezas que permitan la adquisición de formas y estilos de vida saludables de un modo más eficaz (Ortega, 2010).

En lo referente al liderazgo el de tipo transformacional, transaccional y resultados de liderazgo presentaron altas correlaciones entre sí con cifras similares y superiores al de tipo pasivo, por lo que observamos que los resultados muestran también que las correlaciones entre los factores de liderazgo y las variables de resultado organizacional son similares a las señaladas en la literatura (Avolio \& Bass, 2004), contribuyendo así 
a la validación del cuestionario. Por otra parte observamos también que los docentes españoles reciben, por término medio, puntuaciones altas en los factores relacionados con el liderazgo transformacional y transaccional.

Un único estudio no es suficiente para poder interpretar este resultado adecuadamente, ya que este efecto puede deberse, bien a las características específicas de la muestra, bien a una cierta visión predominante en España sobre el liderazgo que conduciría a que un determinado tipo de liderazgo sea mejor evaluado que otro. En este sentido sería importante realizar también investigaciones longitudinales, puesto que la concepción del liderazgo existente en una determinada cultura o sociedad puede variar a lo largo del tiempo (O'Connell, Prieto \& Gutiérrez, 2008).

En cuanto a la enseñanza donde se imparten docencia, la mayoría de los profesores son de Universidad, seguido de los de Primaria y Secundaria, estos datos corroboran los extraídos del Ministerio de Educación, Cultura y Deporte (MECD) en su publicación anual "Datos y cifras" (2017-18) de las enseñanzas no universitarias y del sistema universitario español (MECD, 2017), que tiene por objeto ofrecer los datos estimados al inicio del curso escolar sobre las principales variables del sistema educativo español: alumnado, centros y gasto. Además, se incluyen otras informaciones estadísticas de la educación, así como datos relevantes de la comparativa europea.

No se determinaron diferencias en el caso de la inteligencia emocional en función del tipo de enseñanza, de esta forma, destacar que tradicionalmente siempre se ha considerado a las Enseñanzas Secundarias como característica diferencial en su incidencia en el profesorado por las características psicosociales de este alumnado y las implicaciones que sobre el estrés laboral incidía en este colectivo profesional (Mérida-López \& Extremera, 2017). En la actualidad, y con los programas que se están implementado de formación docente llevados a cabo desde las administraciones educativas sobre la inteligencia emocional y emociones positivas, están llevando al profesor a hacer frente a situaciones difíciles en el aula de manera exitosa, y esta mejora en el ambiente de la clase se está observando en un incremento en la percepción intrapersonal que se interpreta como una mejora en la habilidad del grupo para reconocer conscientemente sus emociones, identificar lo que sienten y ser capaces de ponerle una etiqueta verbal. De la misma manera, un aumento en la percepción interpersonal facilita la capacidad de percibir los estados emocionales de otras personas, así como el comprender cómo estas emociones les afectan a nivel personal, las consecuencias y reacciones que les provocan (Body, Ramos, Recondo \& Pelegrina, 2016).

El aumento en la capacidad de asimilación emocional se interpreta como mejor habilidad para hacer uso de las emociones con el fin de facilitar diferentes procesos cognitivos; por ejemplo, para fijar metas, motivarse o tomar decisiones. Finalmente, los cambios positivos en la regulación emocional representan la habilidad de dirigir y manejar las emociones, tanto positivas como negativas, de forma eficaz (Body et al., 2016).

Si se detectan diferencias, en el caso del liderazgo transformacional, estas proceden de los colectivos de Primaria y Ciclos Formativos que obtienen valores medios inferiores al resto de enseñanzas, esto puede ser debido a la ya instaurada red grupal de los equipos de coordinación del profesorado de éstos tipos de enseñanza con estructuras inmovilistas que imposibilitan el cambio de los líderes en los centros y 
al excesivo trabajo burocrático de los órganos colegiados, por lo que en este sentido gestionar la convivencia de forma participativa exige crear equipos de trabajo que compartan responsabilidades y que estén liderados.

Como se puede observar, el liderazgo pedagógico, transformacional o transaccional, se vuelca sobre el trabajo grupal y destina una parte importante de su energía a crear estructuras participadas por el alumnado y profesorado. Cuando esto no es así, la persona que ejerce el liderazgo acaba asumiendo demasiado trabajo y cansándose de ser un referente solitario para sus compañeros y para el alumnado (Álvarez-Botello et al., 2016).

Como hemos hecho referencia en la introducción, quizá el estudio español más preciso hasta el momento sea el de Murillo y Barrio (1999) con datos de 437 directores de escuelas de Primaria de centros públicos, concertados y privados. En él se constata que el mayor porcentaje de tiempo se invierte en tareas relativas a la administración y la organización de su planificación (36.44\%), el 26.7\% a tareas de liderazgo pedagógico, un $15.6 \%$ a la relación con las madres y padres de los estudiantes, algo menos al desarrollo personal $(14.95 \%)$ y la menor cantidad de tiempo, el 6.31\%, se destina a otras tareas.

Entre las principales limitaciones de este estudio podemos indicar el hecho de que la muestra en algunos estratos es escueta, así como el periodo donde se solicitó la cumplimentación del cuestionario que fue próximo a la finalización del curso.

Como principales conclusiones de este estudio de investigación debemos de señalar el excelente ajuste de estos instrumentos, así como su fiabilidad siendo totalmente adecuadas para el conjunto de docentes de enseñanzas regladas. Dentro del marco de la inteligencia emocional se debe señalar que la regulación y comprensión son las más valoradas y en cuanto al liderazgo los de tipos transformacionales, transaccionales y resultados de liderazgo presentan cifras muy similares. En el ámbito de las relaciones solamente se encontró asociación entre el liderazgo transformacional y el tipo de enseñanza impartida hallándose una mayor capacidad de cambio en el contexto universitario, mientras que en primaria y ciclos formativos la transformación no es tan importante.

Estos datos ponen de manifiesto la necesidad de seguir indagando en esta temática, y plantear actividades de innovación en el marco de la enseñanza reglada.

\section{Referencias}

Álvarez-Botello, J., Torres-Velázquez, A., \& Chaparro-Salinas, E. (2016). Diagnóstico del liderazgo educativo en las Instituciones de Educación Superior del Valle de Toluca. Revista de Investigación Educativa, 34(1). 51-68. doi: http://dx.doi.org/10.6018/rie.34.1.206881 Antonakis, J., \& House, R. (2013). The full-range leadership theory: The way forward. En B. Avolio, y F. Yammarino (Eds.), Transformational and charismatic leadership: The road ahead (pp. 3-33). Ámsterdam: Emerald Group Publishing Limited

Argos, J., \& Ezquerra, P. (2013). Liderazgo y educación. Santander: Editorial de la Universidad de Cantabria.

Avolio, B.J., \& Bass, B.M. (2004). Multifactor Leadership Questionnaire. (3ª ed.). Palo Alto: Mind Garden.

Bass, B., \& Avolio, B. (1999). Manual for the Multifactor Leadership Questionnaire (Form 5X). Redwood City, CA: Mind Garden, Inc.

Bentler, P. M. (1990). Comparative fit indexes in structural models. Psychological Bulletin, 107, 238-246. 
Bisquerra-Alzina, R. (2003). Educación emocional y competencias básicas para la vida. Revista de Investigación Educativa, 21(1), 7-4.

Body, L., Ramos,N., Recondo, O.,\& Pelegrina, M.(2016). Desarrollo de la Inteligencia Emocional a través del Programa Mindfulness para regular las emociones (PINEP) en el profesorado. Revista Interuniversitaria de Formación del Profesorado, 87(30), 47-59.

Bolívar, A., López, J., \& Murillo, F.J. (2013). Liderazgo en las instituciones educativas. Una revisión de líneas de investigación. Revista Fuentes, 14, 15-60. Recuperado de https://revistascientificas.us.es/index.php/fuentes/article/view/2352

Cabello, R., Ruiz, D., \& Fernández, P. (2010). Docentes emocionalmente inteligentes. REIFOP, 13 (1). Recuperado de http://emotional.intelligence.uma.es/documentos/ Docentes_emocionalmente_inteligentes_2010.pdf

Castro, D., \& Tomás, M. (2010). El gobierno y la gestión de la universidad: estudio de los órganos unipersonales. Estudios sobre Educación, 19, 165- 182. Recuperado de https://www.unav.edu/publicaciones/revistas/index.php/estudios-sobre-educacion/ article/view/4604/3972

Cazalla-Luna, N., \& Molero, D. (2014). Inteligencia Emocional Percibida, Ansiedad y Afectos en estudiantes universitarios. Revista Española de Orientación y Psicopedagogía, 25(3), 56-73. doi: https://doi.org/10.5944/reop.vol.25.num.3.2014.13858

Cazalla-Luna, N., \& Molero, D. (2016). Inteligencia emocional percibida, disposición al optimismo-pesimismo, satisfacción vital y personalidad de docentes en su formación inicial. Revista de Investigación Educativa, 34(1), 241-258. doi: http://dx.doi. org/10.6018/rie.34.1.220701

Daly, A., \& Finnigan, K. (2016). Thinking and Acting Systemically: Improving School Districts Under Pressure. Chicago: Amer Educational Research Assn.

Day, C. (2012). New lives of teachers. Teacher Education Quarterly, 39(1), 7-26.

Duarte-Rodríguez, A. (2016). Inteligencia emocional y Gerencia en la organización educativa. In Crescendo. Educación y Humanidades, 3(2), 11-22. doi: http://dx.doi. org/10.21895/in+cres+e.v3i2.1342

Elfers, A., \& Stritikus, T. (2014). How School and District Leaders Support Classroom Teachers' Work With English Language Learners. Educational Administration Quarterly, 50(2), 305-344. doi: 10.1177/0013161X13492797

Estela, A., Gálvez, A., Del Ángel, C., Mejía, E., \& Rodiles, E. (2013). Educación e investigación científica: métodos y estrategias de enseñanza adecuados para lograr que el alumno se motive y desarrolle las competencias que le programa educativo 2012 en México establece en el modelo educativo de nivel secundaria. Revista Iberoamericana para la Investigación y el Desarrollo Educativo, 10. Recuperado de

Frost, D., \& Harris, A. (2003). Teacher leadership: towards a research agenda. Cambridge Journal of Education, 33(3), 479-498.

Goleman, D. (2012). El cerebro y la Inteligencia emocional. Barcelona: Ediciones B.

Hargreaves, A. (2002). Teaching in a box. Emotional geographies of teaching. En C. Sugure \& C. Day (Eds.), Development teachers and teaching practice. International research perspectives. London: Routledge-Falmer.

Hargreaves, A., \& Fullan, M. (2014). Capital professional: transformar la enseñanza en cada escuela. Madrid: Ediciones Morata. 
Harris, A. (2009). Distributed leadership: what we know. En A. Harris (Ed.), Distributed leadership: different perspectives (pp. 11-21). Dordrecht: Springer Netherlands.

Hernández-Castilla, R., Murillo, F.J., \& Hidalgo, N. (2017). Lecciones Aprendidas del Estudio del Liderazgo Escolar Exitoso. Los casos de España en el Proyecto Internacional ISSPP. Revista de Investigación Educativa, 35(2), 499-518. doi: http://dx.doi. org/10.6018/rie.35.2.279241

Hué, C., Esteban, J. L., \& Bardisa, T. (2012). El liderazgo educativo: proyectos de éxito escolar. Madrid: Ministerio de Educación.

Leithwood, K., \& Louis, K.S. (2011). Linking Leadership to Student Learning. San Francisco: Jossey-Bass.

López-Yáñez, J., García-Jiménez, E., Oliva-Rodríguez, N., Moreta-Jurado, B. \& Bellerín, A. (2014). El liderazgo escolar a través del análisis de la actividad diaria de los directores. REICE. Revista Iberoamericana sobre Calidad, Eficacia y Cambio en Educación, 12(5), 61-78. Recuperado de https://revistas.uam.es/index.php/reice/article/view/2828/3045

Lorenzo-Seva, U., \& Ferrando, P. J. (2006). FACTOR: A computer program to fit the exploratory factor analysis model. Behavioral Research Methods, Instruments and Computers, 38(1), 88-91. doi: 10.3758/BF03192753

MacBeath, J., \& Cheng, Y. C. (2008). Leadership for learning: international perspectives. Rotterdam: Sense Publishers.

MacBeath, J., \& Townsend, T. (2011). Thinking and acting both locally and globally: what do we know now and how do we continue to improve? En T. Townsend y J. MacBeath (Eds.), International handbook of leadership for learning (pp. 1237-1254). Dordrecht: Springer Netherlands,

Mayer, J. D., \& Salovey, P. (1997). “What is emotional intelligence? "En P. Salovey y D. Sluyter (Eds.), Emotional development and emotional intelligence: implications for educators (pp.3-31). New York: Basic Books.

McDonald, R. P., \& Marsh, H. W. (1990). Choosing a multivariate model: noncentrality and goodness of fit. Psychological Bulletin, 107, 247-255. doi: http://dx.doi. org/10.1037/0033-2909.107.2.247

MECD (2017). Datos y cifras. Curso escolar 2017-18. Madrid: Secretaria General Técnica.

Mérida-López, S., \& Extremera, N. (2017). Estado de la cuestión sobre inteligencia emocional y burnout en el profesorado por países, año de publicación, ciclos educativos e instrumentos de evaluación. Revista de curriculum y formación del profesorado, 21(2), 371-389.

Molero-Alonso, F., Recio-Saboya, P., \& Cuadrado-Guirado, I. (2010). Liderazgo transformacional y liderazgo transaccional: un análisis de la estructura factorial del Multifactor Leadership Questionnaire (MLQ) en una muestra española. Psicothema, 22(3), 495-501.

Murillo, F.J., \& Barrio, R. (1999). Análisis de la distribución del tiempo de los directivos de centros de enseñanza Primaria. Revista de Educación, 319, 201-222. doi: 10.4438/1988-592X-0034-8082-RE

Murillo, F.J., \& Hernández-Castilla, R. (2015). Liderazgo para el aprendizaje: ¿Qué tareas de los directores y directoras escolares son las que más inciden en el aprendizaje de los estudiantes? Revista electrónica de investigación y evaluación educativa, 21(1), 1-20. doi: 10.7203/relieve.21.1.5015 
O'Connell, J.J., Prieto, J.M., \& Gutiérrez, C. (2008). Managerial Culture and Leadership in Spain. En J.S. Chhokar, F.C. Brodbeck \& R.J. House (Eds.). Culture and Leadership Across the World: The GLOBE book of in-depth Studies of 25 societies (pp. 623-654). Londres: LEA.

Ortega, M. C. (2010). La educación emocional y sus implicaciones en la salud. Revista Española de Orientación y Psicopedagogía, 21(2), 462-470. doi: https://doi.org/10.5944/ reop.vol.21.num.2.2010.11559

Ortega-Álvarez, F., Núñez-Hergueta, E., Molero, D., \& Torres-González, J.A. (2015). Diversidad emocional y satisfacción vital en futuros docentes. Revista nacional e internacional de educación inclusiva, 8(3), 205-217.

Palomo-Vadillo, M. T. (2013). Liderazgo y motivación de equipos de trabajo. Madrid: ESIC Editorial.

Puertas-Molero, P., González-Valero, G., \& Sánchez-Zafra, M. (2017). Influencia de la práctica físico-deportiva sobre la inteligencia emocional de los estudiantes: una revisión sistemática. ESHPA-Education, Sport, Health and Physical Activity, 1(1), 10-24. doi: http://hdl. handle.net/10481/48957

Ritacco, M., \& Bolívar, A. (2016). Impacto del modelo español de dirección escolar en la identidad profesional los líderes escolares. Archivos Analíticos de Políticas Educativas, 24(119), 41-49. doi: http://dx.doi.org/10.14507/epaa.24.2512

Ritacco-Real, M., \& Amores-Fernández, F. (2017). Dirección escolar y liderazgo pedagógico: un análisis de contenido del discurso de los directores de centros educativos en la Comunidad Autónoma de Andalucía (España). Educação e Pesquisa, 5, 1-23. doi: http://dx.doi.org/10.1590/S1678-4634201709162034

Robinson, V. M. (2010). From instructional leadership to leadership capabilities: empirical findings and methodological challenges. Leadership and Policy in Schools, 9(1), 1-26. doi: 10.1080/15700760903026748

Ruiz-Corbella, M. (2013). Liderazgo y responsabilidad educativa: el necesario liderazgo de directores y profesores en la educación. Revista Fuentes, 14, 85-104.

Salovey, P., Mayer, J. D., Goldman, S. L., Turvey, C., \& Palfai, T. P. (1995). Emotional attention, clarity, and repair: exploring emotional intelligence using the Trait MetaMood Scale. En J. W. Pennebaker (Ed.), Emotion, Disclosure, \& Health (pp. 125-151). Washington: American Psychological Association.

Scheerens, J. (2012). School leadership effects revisited. Review and meta-analysis of empirical studies. Dordrecht: Springer.

Schmider, E., Ziegler, M., Danay, E., Beyer, L., \& Bühner, M. (2010). Is it really robust? Reinvestigating the robustness of ANOVA against violations of the normal distribution assumption. Meythodology, 6, 147-151. doi: https://doi.org/10.1027/1614-2241/a000016

Tichy, N. (2003). Líderes en acción. Cómo formar líderes en todos los niveles de una organización. México: Compañía Editorial Continental CECSA.

Zembylas, M., \& Schutz, P. A. (2009). Reserch on teachers' emotions in education: findings, practical implications and future agenda. En P. A. Schutz y M. Zembylas (Eds.), Advances in teacher emotion research: the impact on teachers' lives (pp. 367-377). Boston: Springer.

Fecha de recepción: 2 de noviembre de 2017.

Fecha de revisión: 6 de noviembre de 2017.

Fecha de aceptación: 26 de febrero de 2018. 